Voix et Images

voixetimages

\title{
Jack Kerouac : la feinte et l'écriture
}

\section{Francis Favreau}

Volume 13, numéro 3 (39), printemps 1988

Jack Kerouac et l’imaginaire québécois

URI : https://id.erudit.org/iderudit/200730ar

DOI : https://doi.org/10.7202/200730ar

Aller au sommaire du numéro

\section{Éditeur(s)}

Université du Québec à Montréal

\section{ISSN}

0318-9201 (imprimé)

1705-933X (numérique)

Découvrir la revue

\section{Citer cet article}

Favreau, F. (1988). Jack Kerouac : la feinte et l'écriture. Voix et Images, 13(3), 413-421. https://doi.org/10.7202/200730ar d'utilisation que vous pouvez consulter en ligne.

https://apropos.erudit.org/fr/usagers/politique-dutilisation/ 


\title{
Jack Kerouac: la feinte et l'écriture
}

\section{par Francis Favreau, Université du Québec à Montréal}

\author{
Imaginez - devoir voyager à travers le \\ temps au fil des jours de votre vie avec votre \\ propre visage et le faire ressembler à votre \\ propre visage! \\ Jack Kerouac, les Anges vagabonds, \\ p. 140.
}

Kerouac était un anti-héros, un antipersonnage, il ne s'est jamais cru. C'est dommage et dans un sens c'est parfait: ce sont ceux qui ne se croient pas qui ont le plus de plénitude.

Denis Vanier, Nuit blanche, décembre 1987, p. 34.

Jack Kerouac, malgré lui

Qui était Jack Kerouac? On connaît le mot de François Ricard, «vécriture», pour qualifier ce phénomène d'intrications et de transformations mutuelles entre la vie et l'écriture de Kerouac ${ }^{1}$. Ce dernier se posait lui-même la question de son identité de manière aigué et souvent pathétique, comme lorsqu'il répondit, dans Vanité de Duluoz, à cette lectrice qui lui écrivait: Vous n'êtes pas Jack Kerouac. Jack Kerouac n'existe pas. Personne n'a jamais écrit ses livres 2. L'auteur n'était pas étranger à la confusion de sa lectrice: toute son écriture est ce mouvement par lequel il se gagnait une identité et la perdait. Kerouac-écrivain s'était choisi Kerouac-personnage de ses romans, lequel, à son tour, se choisit comme auteur. Le personnage devient auteur dans la présence d'une écriture qu'il s'approprie.

Est-ce la «vécriture» qui procure ainsi à Kerouac une identité tout en la lui retirant? Où sommes-nous dans son essence même: le mouvement de toutes les identités possibles de Kerouac? Pour le vérifier, nous nous demanderons: $Q u i$, dans la présence fantomatique de «son» écriture, peut être Jack Kerouac?

1 Publié d'abord comme «La "vécriture" de Jack Kerouac» dans Liberté, $\mathrm{n}^{\circ}$ 128, et repris sous le titre "Vécrire» dans la Littérature contre elle-même, Montréal, Boréal Express, coll. «Papiers collés», p. 93-98.

2 Jack Kerouac, Vanité de Duluoz, Paris, Christian Bourgois, 1979, p. 16. 
De fait, Jack Kerouac ne peut être Jack Kerouac que contre la réalité convenue, dépossédante, celle des autres, et même contre soi, dans un chevauchement de ses identités possibles que nous proposons d'appeler la feinte. Comme le souligna William Burroughs, Kerouac a toujours été un écrivain avant tout. Il croyait que tout ce qu'il faisait en tant qu'être humain relevait de la simulation pure et simple 3 . Aussi, la feinte est un terme général pour résumer le paradigme de la simulation, d'un faire-semblant où être le contraire de ce que les autres pensent, être à la place d'un autre, mentir, surprendre, trahir ou utiliser un pseudonyme sont les variations d'une même opposition à une identité fixe et réelle.

Comme le décrit Vanité de Duluoz, la feinte a un sens propre: le mouvement simule au football pour tromper l'adversaire. Ce sens propre est peu important comparé au sens symbolique ou métaphorique. La feinte fut une manière de modus vivendi pour Jack Kerouac: il agit dans la vie comme sur un terrain de football. Seulement, il en laisse un témoignage littéraire. C'est-à-dire que la feinte devient une manière d'organisation rhétorique ou, ce que nous préférons, une métaphore qui donnerait un sens à tous les autres mouvements par lesquels Kerouac rend possible son identité en la simulant. La métaphore de la feinte devrait donc nous permettre de toucher la fiction littéraire dans ce qu'elle a d'originaire, entre la vie et l'écrit.

\section{Une jambe déguisée en crayon}

Dans Vanité de Duluoz, Jack 4 feinte pour échapper aux joueurs de l'équipe de football adverse. Le football semble d'ailleurs être un jeu de feintes perpétuelles pour Jack:

[...] je fonce vers la droite en riant, détale devant leurs bras tendus [...], je change de direction, mais change de direction est une expression trop longue, je saute vers la gauche, prenant tout le monde da contrepied [...] Lebreon plaque un type de Tome, je saute sur la droite [...] Un autre type de Tome. Je saute sur la gauche, le laisse planté là [...] $j$ 'arrive a la ligne des cinq mètres et me retrouve en face de trois types de Tome, [...] mais Jack-le-cerveau saute soudain de nouveau sur la droite, les abandonnant en train de danser le menuet, et nous gagnons ce match 6 a 0 , une autre grosse surprise $[\ldots]^{5}$.

La force de Jack, ce n'est pas son corps trapu, mais son intelligence de la feinte. Jack interprète le football comme l'exercice spontané de sa liberté. C'est elle qu'il éprouve en créant sa propre stratégie, ses propres feintes - car contrairement aux autres joueurs, c'est un créateur:

3 Cité par Ann Charters, Kerouac, le vagabond, Montréal, l'Étincelle, 1975, appendice au chapitre XXXIV, p. 449.

4 Par commodité, tenons-nous en à ce «Jack» qui désigne à la fois le Kerouacauteur et le Kerouac personnage de lui-même, c'est-à-dire Duluoz. Nous verrons plus loin comment ce roman les met en conflit l'un avec l'autre.

5 Vanité de Duluoz, op. cit., p. 60-61. 
[...] ils se préparent d̀ me tomber dessus, c'est psychologique, sans penser une seconde que je leur prépare un petit contrepied que je mets a exécution: je fonce sur la droite et les laisse plantés là d reprendre leur souffle ${ }^{6}$.

S'il abandonne le football à Columbia, c'est parce qu'il n'a plus la liberté de créer ses feintes, son entraîneur Lu Libble lui imposant sa fameuse «KT-79». Jack doit utiliser cette technique de jeu, lui qui jusque-là avait toujours joué d'instinct, spontanément:

[L'entraîneur Lu Libble] m'insulta de nouveau devant tout le monde: "Tu n'es pas vraiment un bon sprinter, et tu ne maîtrises pas le contrepied-supercherie KT-79», - comme si je jouais au football pour la «supercherie» $[\ldots]]$.

Jack joue pour sa propre feinte. Il ne conçoit pas qu'il y en ait une autre que celle qui s'élabore spontanément dans la course, en évitant les adversaires.

Pourtant, s'il veut jouer au football, Jack doit obéir à Lu Libble, même si son autorité dénature l'essence du jeu. Aussi, parce que Lu Libble devient un adversaire contre lequel Jack se croit obligé de feinter, nous assistons à la «métaphorisation» de la feinte. Jack quitte Columbia et l'identité de footballeur qu'elle lui garantissait, en prétextant aller porter des vêtements chez sa grand-mère. En fait, il court s'acheter un billet d'autobus pour le Sud, décidé à commencer sa carrière de vagabond américain 8 .

Ce qu'il faut comprendre, c'est que le football en soi était déjà une feinte pour Jack. Son costume de footballeur a été l'un de ses premiers déguisements. Il y cachait une ambition d'écrire: [...] j'allai d Columbia car je désirais connaître New York et devenir un célèbre journaliste sur la place de cette ville célèbre9. Jack ne veut pas être celui que l'on croit. La feinte en tant que mouvement de ses identités possibles lui permet de jouer sur son nom, sur son autre nom, et de se comparer au Témoin des Écritures: Qui donc avait pu battre St. John [une équipe adverse]? Eh bien Saint-Jean Duluoz et ses potes, pardi. [...] il faut bien avoir un Saint-Jean dans son équipe $e^{10}$. Le football, c'était pour écrire. Mais pourquoi devenir un «vagabond américain»?

En se cassant la jambe, Jack a vu se briser ses espoirs de footballeur (pour cette année-là du moins), et ses projets d'écriture qui y étaient associés:

[...] c'était une jambe cassée, fracture du tibia, comme si tu cassais un os a peu près gros comme un crayon: le crayon reste entier, d̀

$6 \quad$ Ibid., p. 86.

7 Ibid., p. 112. Il s'agit bien sûr de Lou Little.

8 Ibid., p. 114.

9 Ibid., p. 90.

10 Ibid., p. 56-57. 
l'exception d'une fissure mince comme un cheveu, si bien que tu peux casser le crayon (si tu le désires) rien qu'avec deux doigts ${ }^{11}$.

Plus de jambe, plus de moyen pour écrire suggère la comparaison, et c'est sûrement ainsi que le ressentit d'abord Jack. Toutefois, durant son immobilisation, il découvre Tom Wolfe qui élargit pour lui le terrain de jeu de l'écriture aux dimensions d'un continent:

\section{[...] il m'apprit tout simplement d voir l'Amérique comme un Poème et non seulement comme un lieu où se battre et baver. Ce poète améri- cain aux yeux sombres me donna l'envie de rôder, d'errer, de voir l'Amérique réelle, sans fard, celle «qui n'avait jamais été dite»12.}

Grâce à Wolfe, Jack conserve intacte, et même renouvelle sa puissance de voir et de témoigner: l'Amérique «réelle» attend son Poète. Quelle excellente raison pour tourner le dos à un stade de football limité, mesuré, pour devenir «vagabond américain»: quelqu'un qui n'a plus d'identitê aux yeux des autres parce qu'il est emporté dans le mouvement de toutes ses identités possibles...

Mais quelle forme le Poème de l'Amérique peut-il prendre?

\section{Le Docteur Sax, cette rivière}

L'œuvre de Kerouac est protéiforme. Comment deviner que Visions de Gérard et Docteur Sax proviennent de la même plume tant leurs styles diffèrent ${ }^{13}$ ? Ils diffèrent même peut-être autant parce que l'écriture qui demeure spontanée 14 l'exige. Ainsi, on ne peut pas considérer les œuvres précédant la période «beat» ( et sa meilleure expression: Sur la route) comme des tentatives de style. Dans son écriture spontanée, chaque roman est une forme de la Forme kaléidoscopique du Poème de l'Amérique.

Pour Kerouac, en autant qu'elle l'identifiait à une écriture ( la «sienne»), cette spontanéité a été possible dans la feinte qu'elle représente: ce changement de forme à chaque roman. Cette feinte n'est pas dirigée directement contre les lecteurs, mais contre une institution littéraire et surtout contre Kerouac lui-même, contre sa propre existence, pour qu'elle accède à la fiction et qu'il la perçoive, dans l'hétérogénéité de ses formes, comme transitoire. Le terme bien sûr réfêre au «saxisme» et au bouddhisme de Kerouac; tout est passager, provisoire comme les multiples attitudes du Docteur Sax.

\section{Ibid., p.88.}

12 Ibid., p. 92. Nous soulignons.

13 Voir aussi Maggie Cassidy, disons, par rapport à Visions de Cody. Sur cette question d'expérimentation des formes, on devrait consulter l'ouvrage récent de Regina Weinreich, The Spontaneous Poetics of Jack Kerouac: A Study of the Fiction, Carbondale \& Edwardsville (Ill.), Southern Illinois University. Press, 1987.

14 Kerouac s'explique lui-même sur «l'écriture spontanée» de ses livres. 
Le Docteur Sax est peut-être, mis à part Jack lui-même, le personnage le plus important de Kerouac, parce que contrairement à d'autres, le lecteur le saisit comme pure fiction: c'est un produit fantasmatique de l'imagination de Jack, luimême personnage. Le Docteur Sax est un personnage en mouvement, «bougé», différent de Moriarty qui parcourt le continent, car son mouvement appartient à la métamorphose, à la perte d'identité, à la feinte - comme personnage «fictif» (fantasmé), c'est un mouvement d'écriture qui décide de son identité.

Jack a besoin d'un miroir pour se voir devenir écrivain avant de l'être; Jack utilise Sax pour se croire Kerouac l'écrivain. Il a besoin de cet homme de savoir, ce Docteur dont il ne sait rien, pour approcher l'origine de toutes les identités possibles: Sax indique à Jack l'accès du monde souterrain, ce monde de la métamorphose printanière ${ }^{15}$. C'est un monde en lutte pour l'émergence originelle d'une Forme, le Serpent, symbole de la feinte, du monde illusoire et destructeur de la Maya et de Satan. En outre, la lutte entre Sax et le Serpent en train de naître à sa forme est aussi la lutte de l'écriture, du livre Docteur Sax: le Serpent destructeur disparu, Sax cesse lui-même de symboliser la feinte originelle et créatrice, il redevient un homme, le livre se termine.

Kerouac a eu besoin de l'apprentissage de Jack auprès de Sax pour écrire, car le personnage fantasmé de Sax est une sorte de mise en abyme, de degré originaire de la fiction. Sax est un mouvement créateur qui embrasse toutes les identités possibles. Jack s'initie avec lui au «saxisme» de l'existence, c'est-à-dire à une manière de contrôler son identité pour feinter puisque l'identité en soi est considérée comme une feinte. Pour Jack, deviennent «saxistes» les jeux d'enfance avec Gene Plouffe déguisé en «Homme-lune», ou son propre déguisement en «Bandit noir» pour apeurer Dicky. En fait, et c'est important, le contrôle «saxiste» de l'identité s'exerce sur l'origine des identités possibles, sur la création de l'identité.

Nous pouvons donc parler d'un «saxisme» de l'écriture kérouacienne, inattendu et surprenant, «jazzé», pareil à la rivière Merrimac qui fond en mars et inonde la ville natale de Jack: Tout au fond de moi, je suis conscient de l'action de la rivière, les mots glissent et serpentent lentement comme la rivière et quelquefois débordent... 16. L'eau devient écriture et l'écriture se fait eau; l'écriture est une perte d'identité comme l'eau-Serpent de la feinte. L'une et l'autre jouent l'ambivalence: créatrices et destructrices à la fois; destructrices parce que créatrices. Dans Docteur Sax, la formation de Jack est mise en scène dans un style chaotique et spontané qui est lui-même une formation en devenir. L'eau comme l'écriture, dans ce chaos thématique et stylistique, sont le Serpent destructeur, mais tout aussi bien Docteur Sax créateur; la confusion est telle que l'un et l'autre échangent leur identité, car si l'eau est le Serpent, elle est aussi Sax qui le

15 On nous excusera de ne pas aborder ici la question du «sexe», thème évident du récit.

16 Jack Kerouac, Docteur Sax, Paris Gallimard, coll. «Du monde entier», 1972 , p. 180. 
combat: [...] j'eus l'occasion de faire le rapprochement entre le Docteur Sax et une riviere $[. . .]^{17}$.

Il n'y aurait pas d'écriture sans liberté, sans spontanéité, les mêmes que l'eau qui soudain inonde tout. Sax est une rivière en métamorphose, un mouvement de création parfois bien, parfois mal, qui connaît l'origine de ses identités possibles: le sous-sol du Serpent: [...]' et là, énorme et primordial, jaillit le fleuve issu de la terre qui poussait sa course dévorante vers la mer monstrueuse ${ }^{18}$.La terre engendre la feinte. Du sous-sol, l'eau et l'écriture jaillissent pour tout recouvrir.

L'ambivalence de l'eau et de l'écriture, à la fois origine et destruction, bien et mal, est peut-être étrangère au «saxisme» comme tel. Non que le «saxisme» ne soit porteur d'ambivalence, mais il est du côté de la création contre le Serpent destructeur (en autant qu'on se permette des catégories aussi tranchées) - le «saxisme» s'oppose à la «vanité», et ce sont ces deux mouvements qui font l'ambivalence de l'eau et de l'écriture.

Dans Vanité de Duluoz, cette ambivalence se manifeste une dernière fois. Lorsque Claude de Maubris tue Franz Mueller, il jette son cadavre dans l'eau de la rivière Hudson. Les pièces à conviction (ou ce qui pourrait en tenir lieu) vont toutes dans le sous-sol, comme retournant à l'eau originelle de la feinte: les lunettes dans la terre, le canif dans un conduit de métro, le blouson taché de sang dans un égoût. Et tout cela est devenu de l'écriture, tout cela a rejailli du sol comme si la création littéraire annulait la destruction d'un être humain. Mais dans ce dernier livre, Kerouac se détruit et il appelle la création une "vanité» Sax n'est qu'un tas de mots inanimés pour Kerouac au seuil de sa mort.

N'est-ce pas étonnant que vers la fin de ce livre, il raconte la mort de son père? Comment le lire? Du moins, l'eau est encore présente dans toute son ambivalence, car le père de Jack meurt l'estomac plein d'eau qu'il aura fallu, en vain, drainer. Cette mort révèle à la fois une «vanité» tragique et un «saxisme», une feinte où Jack promet à son père de s'occuper de sa mère en prenant, enfin, toute la place auprès d'elle. Le père est mort, vive le père. Mais Kerouac écrit sur la mort de son père parce qu'elle est une prédiction de la sienne, toute proche, qui transfigure la vie qui reste à vivre en vanité. Qu'en est-il plus précisément de la vanité dans ses liens avec le père? En quoi est-elle un aspect de la feinte?

\section{La place dans l'autre}

La feinte est le support d'une ambivalence de l'origine et de la mort, d'une identité qu'elle donne et enlève à Jack. Par exemple, à l'origine de la vie de Kerouac, il y eut la mort de son frère. Mais Gérard mort, l'écriture le ressuscite - aussi est-ce un bien qu'il soit mort. Kerouac, par la feinte de l'écriture, «déguise» Jack en Gérard pour qu'il en prenne la place, pour qu'il dépasse enfin le modele:

17 Ibid., p. 40.

18 Ibid., p. 181. Nous soulignons. 
Pendant les quatre premières années de ma vie, tant qu'il vécut, je ne fus pas Ti-Jean Duluoz, je fus Gérard [...] Pour moi, les quatre premières années de ma vie sont imprégnées [...] par le souvenir d'un visage doux et sérieux qui se penche au-dessus de moi, qui est moi et qui me bénit ${ }^{19}$.

La feinte est remarquable: Jack (Ti-Jean) devient une de ses identités possibles, Gérard, quand Gérard lui-même devient Jack: Qui naît, qui meurt finalement dans cette gémellité?

La feinte comme mouvement ambivalent d'identités va aussi pousser Jack à contourner l'autorité de son père. Ce dernier s'oppose à ce qu'il devienne écrivain: Jamais un Duluoz n'est devenu un grand écrivain... jamais eu un nom qui ressemble à ça dans cette branche 20 . Le nom propre devient le centre du problème. Le père est le propriétaire du nom propre et il refuse à Jack l'espoir d'un nom d'écrivain. N'est-ce pas une feinte contre le père que Kerouac effectue en prenant le nom de Duluoz dans ses romans? Par là, il donne même un nom à son père - il en est l'égal quant au contrôle de l'origine. Mais ce n'est pas suffisant, car Kerouac finit par considérer Duluoz comme une vanité.

On sait que Kerouac occupe de manière œdipienne la place du père auprès de sa mère. Ses histoires d'amour sont chaque fois des trahisons et des feintes envers la femme aimée afin que ne soit pas trahi son Amour pour la Femme, Mémère. On peut évoquer Mardou (les Souterrains), Tristessa ou Terry (la Mexicaine de Sur la route); chaque fois, s'il restait auprès de ces femmes, Kerouac laisserait vide la place du père.

Kerouac tenta bien de contourner le problème de son nom d'écrivain en devenant journaliste au Sun de Lowell. Son père, linotypiste au même journal, en était très fier. Pour Jack, écrire le plaçait avant son père dans la chaîne de production du journal, avant l'autorité qui décidait qui il était. Malheureusement, cette feinte contre l'autorité paternelle échoua, comptant pour une vanité parmi celles qui font toute la matière de Vanité de Duluoz: la fuite de Columbia, du Sun, de la marine marchande, de la Navy. Dans ce roman, Jack devait épuiser une identité après l'autre parce qu'il n'a pas un nom d'écrivain, parce que le père demeure le plus fort. Jamais, même au terme de l'œuvre entière, le père ne fut éliminé symboliquement. Là-dessus, Victor-Lévy Beaulieu observe très finement:

Mais le père devait mourir bien avant la publication de The Town and the City [premier livre de Kerouac] et je crois que Jack ne s'en est jamais vraiment remis puisqu'il n'avait pu faire sa preuve, donc vaincre le Père21.

19 Jack Kerouac, Visions de Gérard, Paris, Gallimard, coll. «Du monde entier», 1972, p. 8-9.

20 Vanité de Duluoz, op. cit., p. 117.

21 Victor-Lévy Beaulieu, Jack Kerouac: essai-poulet, Montréal, Editions du 
Le père, même le père Duluoz, a raișon: Kerouac n'a jamais pu lui montrer qu'il avait un nom d'écrivain.

Avec Vanité de Duluoz, Kerouac fait le constat de son échec: la création, le «saxisme», Duluoz, sa «légende», tout ce qui est issu de la feinte est vanité. La feinte est démasquée, donnée pour ce qu'elle est. Kerouac rature son personnage qui le rature à son tour; si on veut, c'est une sorte de suicide par personnage interposé. Kerouac et Duluoz ne sont pas parvenus à se confondre, la réalité du nom de Kerouac a été la plus forte et la feinte, le «saxisme» sont de dangereuses sources de folie. Il faut renoncer à la feinte, sinon

[...] jen viendrai d̀ penser que je ne suis pas du tout Jack Kerouac, que mes actes de naissance, ceux de ma famille, mon arbre généalogique, mes résultats sportifs [...], mes propres calepins et livres publiés, ne sont pas réels du tout, mais un tas de mensonges, et même que mes propres rêves rêvés dans mon sommeil nocturne ne sont pas des rêves du tout, mais les inventions de mon imagination éveillée, que ne ne suis pas «je», mais simplement un espion dans le corps de quelqu'un d'autre qui prétend que je suis un éléphant traversant Istanbul avec les indigènes à ses trousses 22 .

La réalité doit vaincre la fiction. C'est pourquoi durant tout ce roman, Jack ne cesse d'interpeller la lectrice la plus réelle qu'il ait, sa femme...

Mais la réalité la plus crue, c'est la vanité et la mort qui la révèlent à Kerouac. Quel autre PECHÉ existe-t-il sinon celui de la naissance? ${ }^{23}$ demandet-il à bout de feintes, de fictions, et renonçant à occuper la place d'un autre, renonçant au mouvement de ses identités possibles.

Nous pourrions poursuivre encore l'examen du déploiement métaphorique de la feinte dans l'œuvre kérouacienne, mais nous en avons vu les éléments principaux. Nous nous étions demandé au départ: Qui peut être, dans la présence de «son» écriture, Jack Kerouac? Une telle question impliquait une évaluation des identités possibles de Kerouac et de leur mouvement dans son œuvre.

Nous avons interprété le «saxisme» et la «vanité» complémentaires pour qu'il y ait un mouvement de la feinte. Mais ce mouvement, dès lors, devait être considéré ambivalent, c'est-à-dire constitué des valeurs de l'origine et de la mort. Enfin, ce mouvement ambivalent semble limiter les identités possibles en limitant l'écriture elle-même. Dans sa souffrance, Kerouac a su mieux qu'un autre

Jour, 1972, p. 77.

22 Vanité de Duluoz, op. cit., p. 17.

23 Ibid., p. 324. 
transmettre cette épreuve si individuelle des limites humaines, jusqu'à nous montrer naître puis mourir en lui la création. En ce sens, il aura été plus qu'un Témoin de la passion d'un autre, il aura été son propre Témoin.

Par là, questionnant l'origine de la fiction, la feinte se montre une dimension essentielle de la "vécriture». Bien que le rôle du père ait été abordé, il eût fallu parler, pour être exhaustif, de celui de la mère. Quelle a été son action dans l'écriture de ce Poème wolfien de l'Amérique? De même, quels sont plus précisément les apports du bouddhisme, du «jésuitisme», des drogues dont nous n'avons pas parlé? Vers quelle folie 24 d'être tous ses personnages entraînaient-ils Kerouac?

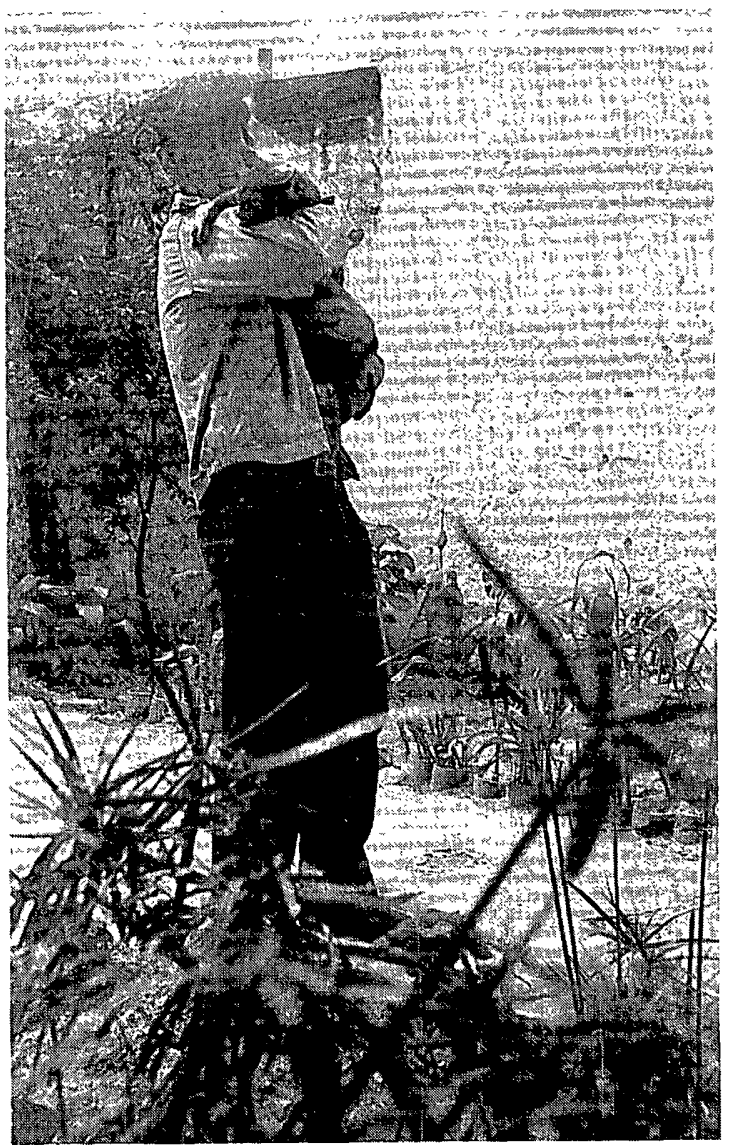

24 Dans l'œuvre de Kerouac, l'expérience de la folie se concentre surtout dans Big Sur. Là, «Jack» ne s'identifie qu'à lui-même, un peu à la manière de Dr. Jekyll et Mr. Hyde, roman qui est mentionné dans le récit et qui sert de «feinte» intertextuelle. 